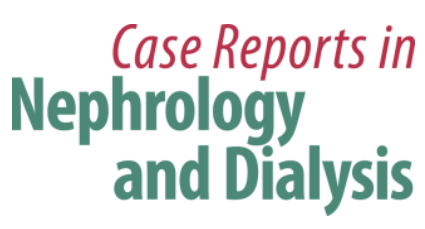

\title{
Refractory Abdominal Pain in a Hemodialysis Patient
}

\author{
Ying Qian Xiao-nong Chen Hao Shi Jingyuan Xie Nan Chen \\ Department of Nephrology, Ruijin Hospital Affiliated to Shanghai Jiaotong University \\ School of Medicine, Shanghai, China
}

\section{Key Words}

Nonocclusive mesenteric ischemia $\cdot$ Hemodialysis $\cdot \mathrm{CT}$ angiography

\begin{abstract}
Nonocclusive mesenteric ischemia (NOMI) is a rare disorder. Failure of an early diagnosis may cause progressive intestinal ischemia, leading to abdominal pain, sepsis, and death. Patients with end-stage renal disease are among the highest risk populations for developing this lethal complication. The key to a correct diagnosis at an early stage is a high index of suspicion in predisposed patients. In our case, we present a 62-year-old female undergoing maintenance hemodialysis for 8 years; she complained of abdominal pain after hemodialysis in the last 3 months; NOMI was suspected after a CT angiography. She partially recovered after multiple clinical interventions such as decreased ultrafiltration, an increased dose of low molecular-weight heparin and the use of vasoactive drugs. In conclusion, NOMI can be reversible if it is diagnosed as early as possible and after the necessary diagnostic measurements are initiated.

(C) 2015 S. Karger AG, Basel
\end{abstract}

\section{Introduction}

Mesenteric ischemia without thromboembolic occlusions of the mesenteric arteries is known as nonocclusive mesenteric ischemia (NOMI). Current knowledge of the epidemiology of NOMI is limited and mainly derived from published cases [1-4]. The dialysis population, and in particular hemodialysis (HD) patients, are especially prone to develop NOMI [5]. The estimated incidence of mesenteric ischemia in this group is in the range of $0.3-1.9 \%$ per patient/year, in contrast to an up to $0.2 \%$ value for the general population. The incidence appears to be increasing steadily during these years. This could be due in part to the increasing number of published cases as well as the increasing survival of end-stage renal disease

KARGER 125/s $\begin{aligned} & \text { Nan Chen } \\ & \text { Department of Nephrology } \\ & \text { Ruijin Hospital Affiliated to Shanghai Jiaotong University School of Medicine } \\ & 197 \text { Ruijin Road, Shanghai 200025 (China) } \\ & \text { E-Mail Chen-nan @ medmail.com.cn }\end{aligned}$




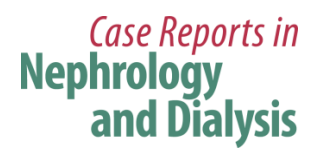

Case Rep Nephrol Dial 2015;5:145-151

DOI: 10.1159/000431084

c) 2015 S. Karger AG, Basel

www.karger.com/cnd

Qian et al.: Refractory Abdominal Pain in a Hemodialysis Patient

(ESRD) patients, predisposing them to NOMI [6]. In the absence of noninvasive tools with sufficient sensitivity and specificity, increased awareness and early diagnosis might improve the general understanding of the NOMI incidence and of the characteristics of patients with NOMI.

\section{Case Presentation}

We present a 62-year-old female with ESRD due to hypertension. She commenced maintenance HD in October 2001. Her past medical history included primary hypertension, paroxysmal atrial fibrillation, atherosclerosis of the carotids and the abdominal aorta, and chronic congestive heart failure. The procedure of HD was 3 times per week and $4 \mathrm{~h}$ each time. Her body weight was $46 \mathrm{~kg}$ and the ultrafiltration volume was around $2.5-2.7 \mathrm{~kg}$ each time. During HD, the occurrence of hypotension was frequent. The blood pressure was $120 / 70 \mathrm{~mm} \mathrm{Hg}$ before HD and dropped to $80 / 60 \mathrm{~mm} \mathrm{Hg}$ after HD. She became anuric after maintenance HD for 2 years. In August of 2008, she was admitted to our department because she developed mild, diffuse abdominal pain in combination with nausea and vomiting. Three months prior to admission, she had several episodes of abdominal pain, each time occurring after HD. Her abdominal pain began and became more severe after HD (usually in the situation of hypotension). However, repeated blood tests did not show any abnormal results and an abdominal X-ray ultrasound was also normal. On admission, her physical examination showed that her blood pressure was $120 / 70 \mathrm{~mm} \mathrm{Hg}$, a heart rate of 76 beats/min and a body weight of $43 \mathrm{~kg}$; she had dry skin and complained of thirst. An abdominal examination revealed that she had decreased bowel sounds with a mild tenderness to deep palpation, especially in the lower right quadrants. A stool examination for occult blood was negative. Laboratory findings included blood urea $30.9 \mathrm{mmol} / \mathrm{l}$, serum creatinine $749 \mathrm{umol} / \mathrm{l}$, serum amylase $34 \mathrm{IU} / \mathrm{l}$, calcium $2.2 \mathrm{mmol} / \mathrm{l}$, phosphate $2.3 \mathrm{mmol} / \mathrm{l}$, albumin $30 \mathrm{~g} / \mathrm{l}, \mathrm{iPTH} 130 \mathrm{pg} / \mathrm{ml}$, hemoglobin $114 \mathrm{~g} / \mathrm{l}$, and a white blood cell count of $11.1 \times 10^{9} / \mathrm{l}$. The rest of her laboratory examination (including disseminated intravascular coagulation, C-reactive protein, erythrocyte sedimentation rate, and tumor biomarkers) was within normal limits. Stool cultures for bacteria, fungus and tubercle bacillus were all negative. The echo examination showed that her left ventricular ejection fraction was $56 \%$. A plain abdominal film in the erect position disclosed abdominal distention with small fluid levels of the small intestine. No free air and mass were noticed anywhere in the peritoneal cavity. An enhancement CT showed intestinal dilatation indicating a mesenteric ischemia; there was no evidence of a tumor, severe intestinal infection or obstruction. The patient was initially treated with intravenous antibiotics (rocephin $2.0 \mathrm{~g}$ once daily). Her medical condition did not improve in the following days. A CT angiography (CTA) was then performed, and the result showed that she had a portal vein thrombus with a normal superior mesenteric vein and a mesenteric artery stenosis suspecting NOMI (fig. 1, fig. 2, fig. 3, fig. 4). After her CTA examination, she was treated with subcutaneous low-molecular-weight heparin (fraxiparine 5000 anti-Xa IU/ml, subcutaneously once and twice every other day). The patient refused to undergo an endoscopic examination. Thereafter, we recommended decreasing her ultrafiltration volume to less than $2 \mathrm{~kg}$ each time in order to avoid episodes of hypotension during or after HD; she was treated with intravenous prostaglandin E1 $10 \mu \mathrm{g} /$ day, and the abdominal pain gradually declined after 2 weeks. She was later discharged. 


\section{Case Reports in \\ Nephrology \\ and Dialysis}

Case Rep Nephrol Dial 2015;5:145-151

DOI: $10.1159 / 000431084$

2015 S. Karger AG, Basel

www.karger.com/cnd

Qian et al.: Refractory Abdominal Pain in a Hemodialysis Patient

\section{Discussion}

NOMI, first described by Ende in 1958 [7], is one of the diseases that affect the vasculature of the intestine. The causes of mesenteric ischemia have been traditionally classified as either occlusive or nonocclusive, based on the underlying pathology. Occlusive causes of mesenteric ischemia comprise about $80 \%$ of all cases and include acute mesenteric arterial thrombosis or embolism, chronic mesenteric ischemia, mesenteric venous thrombosis, and strangulated bowel obstruction. Most studies have shown that $20 \%$ of all cases of mesenteric ischemia are nonocclusive in origin [8,9]. The overall incidence rate of NOMI is estimated to be approximately $1 / 5,000$ in hospitalized patients [9]. The mortality rate, despite a decrease from a shocking $80 \% 20$ years ago, is still high, at approximately $50 \%$. A major reason for this high mortality rate is delayed diagnosis and treatment initiation, which may range from $12 \mathrm{~h}$ to 11 days [10-12]. In clinical practice, the majority of patients die from NOMI without any suspicion of intestinal infarction, even at the time of the patient's death. The principal pathophysiology for NOMI is still not completely clear. High-risk factors include atherosclerosis, low cardiac output states (recent myocardial infarction, congestive heart failure, and arrhythmias), ESRD and medications that are known to reduce mesenteric blood flow; for example, cyclosporine, propranolol and phenobarbital overdose, ergot derivatives. Other causes of mesenteric vasospasms are various forms of shock, septicemia, dehydration, and hypotension following heart surgery (cardiopulmonary bypass) or major abdominal surgery.

The dialysis population, and in particular hemodialysis patients, are especially prone to the development of NOMI. The estimated incidence of mesenteric ischemia in this group is in the range of $0.3-1.9 \%$ per patient/year, in contrast to an up to $0.2 \%$ value for the general population. Incidence rates appear to be increasing steadily during these years. This could be because of an increased number of published cases as well as the increased survival of ESRD patients, predisposing them to NOMI. The susceptibility of dialysis patients to NOMI is explained by the common known risk factors in this patient group: widespread atherosclerosis, advanced age, long-standing hypertension, diabetes mellitus, congestive heart failure, arrhythmias, and the use of mesenteric vasoconstrictor drugs are common [13, 14]. In addition, ESRD patients suffer from so-called calciphylaxis by the presence of inappropriately high calcium-phosphorus product because of the routine use of calcium-based phosphate binders and high-dose vitamin $\mathrm{D}$ analogues, resulting in the calcification of the intima and media layers of their arteries [14]. Moreover, the use of recombinant human erythropoietin in HD patients also plays a key role in the occurrence of NOMI. Recent studies in an in vitro and ex vivo model demonstrated that erythropoietin also has a direct vasopressor effect on small mesenteric resistance vessels [15]. Finally, hypoalbuminemia has also been considered a risk factor. Hypotension, especially when there are repeated episodes, is the most important and immediate precipitating factor for NOMI in dialysis patients. In our case, the patient has accelerated atherosclerosis, arrhythmias, hypoalbuminemia and hyperphosphatemia, frequent hypotension during the HD, her body weight on admission was $43 \mathrm{~kg}$ (which means ca. $23.5 \mathrm{l}$ of body water) and her ultrafiltration was $2.5-2.7 \mathrm{~kg}$ (which meant the removal of more than $10 \%$ of total body water); thus, it can definitely provoke ischemia. The condition improved after rehydration by decreasing ultrafiltration. It seems that the combination of nondistensible, calcified mesenteric blood vessels and a dialysis-induced hypotension is the background upon which splanchnic hypoperfusion is liable to occur.

The clinical manifestation of NOMI is variable, depending on the length of the involved bowel, the rapidity of hypoxia onset, and the intrinsic resistance of the bowel wall to ischemia and bacterial penetration. When present, pain is usually severe, but it may vary in intensity, character, and location. Usually it appears 8-12 $\mathrm{h}$ after HD, but it may also appear dur- 


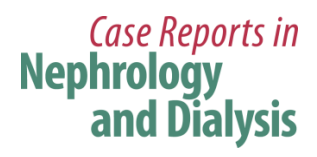

\begin{tabular}{l|l}
\hline \multicolumn{2}{l}{ Case Rep Nephrol Dial 2015;5:145-151 } \\
\hline DOI: 10.1159/000431084 & $\begin{array}{l}\text { @ 2015 S. Karger AG, Basel } \\
\text { www.karger.com/cnd }\end{array}$ \\
\hline
\end{tabular}

Qian et al.: Refractory Abdominal Pain in a Hemodialysis Patient

ing the course of it. The most common symptom is the initiation of the right lower quadrant pain that progresses to peritoneal signs and guarding. Just like in our case, the pain was localized in the right abdomen. Since the pattern of intestinal involvement in NOMI is predominantly right, the cecum and the right colon are most commonly affected. In the absence of pain, unexplained abdominal distention and gastrointestinal bleeding may be the earliest signs of ischemia and impending intestinal infarction. Fever, ileus, and diarrhea are common, but nonspecific manifestations; however, this triple association along with leukocytosis strongly suggests intestinal ischemia. Diffuse or localized abdominal tenderness, rebound and rigidity are ominous signs and usually herald transmural bowel infarction and peritonitis. Although studies have described a high incidence of abdominal guarding upon presentation, mild abdominal signs do not rule out this diagnosis. On the contrary, the finding of a 'nonsurgical' abdomen when faced with a hypotensive HD patient with severe abdominal pain, especially in the right iliac fossa, should alert the nephrologist to the very real possibility of NOMI. The nephrologist should keep in mind not to dialyze their patients too much; they could induce dehydration and accurately estimating their lean body weight. In our case, we estimated the lean body weight only by the clinical observation of the blood pressure and cerebrovascular disease symptoms; the finding of v. portae thrombosis [which might be of the same ethiology (dehydration)]. Therefore, it is very important to assess the proper lean body weight in HD patients with several high risk factors of dehydration such as low heart output and malnutrition. With some help of available techniques like vena cava inferior diameter or bioimpedance analysis, which of course are not $100 \%$ reliable, the more accurate assessment of the lean body weight should be performed in predisposed patients.

Hematologic and serologic abnormalities are nonspecific. Plain abdominal X-rays are useful in excluding other causes of abdominal pain, such as perforated viscus or bowel obstruction. A normal plain film in a patient with pain out of proportion to physical findings is suggestive of early acute mesenteric ischemia and should prompt the consideration of diagnostic CTA and Doppler echography.

In conclusion, despite its rarity, NOMI should always be suspected in the differential diagnosis of abdominal pain in HD patients. The diagnosis of NOMI should be strongly considered in every patient who has abdominal pain and risk factors associated with the development of dehydration, proper dry weight assessment and ultrafiltration prescription in vulnerable populations are 2 keys to avoid and relive the occurrence of NOMI.

\section{Disclosure Statement}

On behalf of Dr. Chen Nan and the other authors, we declare that no financial or other conflict of interest exists in relation to the content of the article.

\section{References}

1 Endean E, Barnes S, Kwolek C, Minion D, Schwarcz TH, Mentzer R: Surgical management of thrombotic acute intestinal ischaemia. Ann Surg 2001;6:801-808.

2 Edwards M, Cherr G, Craven T, et al: Acute occlusive mesenteric ischaemia: surgical management and outcomes. Ann Vasc Surg 2003;17:72-79.

3 Park W, Glovicki P, Cherry K, et al: Contemporary management of acute mesenteric ischemia: factors associated with survival. J Vasc Surg 2002;35:445-452.

4 Acosta S, Ögren M, Sternby NH, Bergqvist D, Björck M: Fatal nonocclusive mesenteric ischaemia: populationbased incidence and risk factors. J Intern Med 2006;259:305-313. 
Qian et al.: Refractory Abdominal Pain in a Hemodialysis Patient

5 Zeier M, Hupp T, Wiesel M, Rambausek M, Ritz E: Nonocclusive intestinal ischemia as a complication of hemodialysis treatment. Dtsch Med Wochenschr 1993;118:1020-1024.

6 Bassilios N, Menoyo V, Berger A, Mamzer MF, Daniel F, Cluzel P, et al: Mesenteric ischaemia in haemodialysis patients: a case/control study. Nephrol Dial Transplant 2003;18:911-917.

7 Ende N: Infarction of the bowel in cardiac failure. N Engl J Med 1958;258:879-881.

8 Haglund U, Lundgren O: Non-occlusive acute intestinal vascular failure. Br J Surg 1979;66:155-158.

9 Liu HL, Huang JJ, Lan RR, Wang MC, Sung JM, Hsieh RY: Ischaemic bowel disease in patients on continuous ambulatory peritoneal dialysis. Nephrol Dial Transplant 1999;14:2032-2034.

10 Williams LF: Mesenteric ischemia. Surg Clin North Am 1988;68:331-353.

11 Dahlberg PJ, Kisken WA, Newcomer KL, Yutuc WR: Mesenteric ischemia in chronic dialysis patients. Am J Nephrol 1985;5:327-332.

12 Stoney RJ, Cunningham CG: Acute mesenteric ischemia. Surgery 1993;114:489-490.

13 Erdberg A, Korzets Z, Neufeld D, Rathaus M, Cordoba M, Bernheim J: Malignant hypertension: a possible precursor to the future development of mesenteric ischaemia in chronically haemodialysed patients. Nephrol Dial Transplant 1992;7:541-544.

14 Ori Y, Chagnac A, Schwartz A, Herman M, Weinstein T, Zevin D, et al: Non-occlusive mesenteric ischemia in chronically dialyzed patients: a disease with multiple risk factors. Nephron Clin Pract 2005;101:c87-c93.

15 Heidenreich S, Rahn K-H, Zidek W: Direct vasopressor effect of recombinant human erythropoietin on renal resistance vessels. Kidney Int 1991;39:259-265.

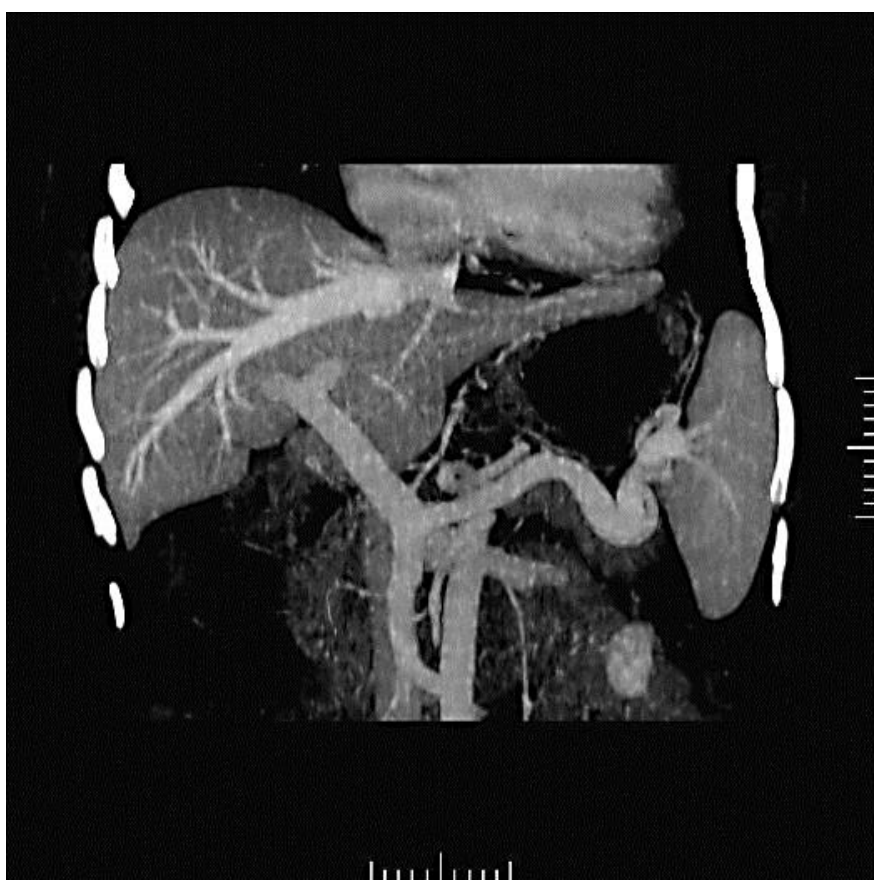

Fig. 1. A CTA showed a normal vasculature of the intestine in 2005. 


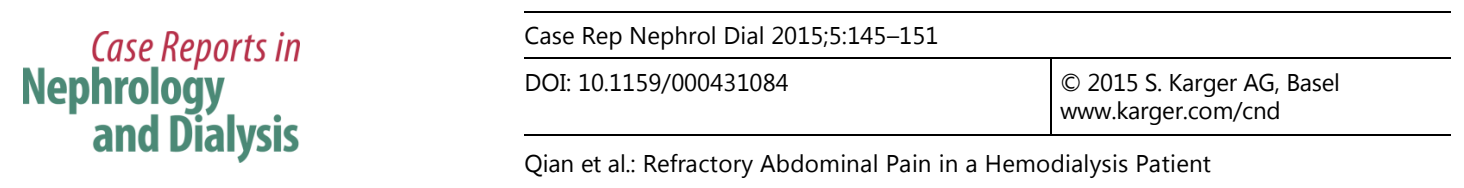

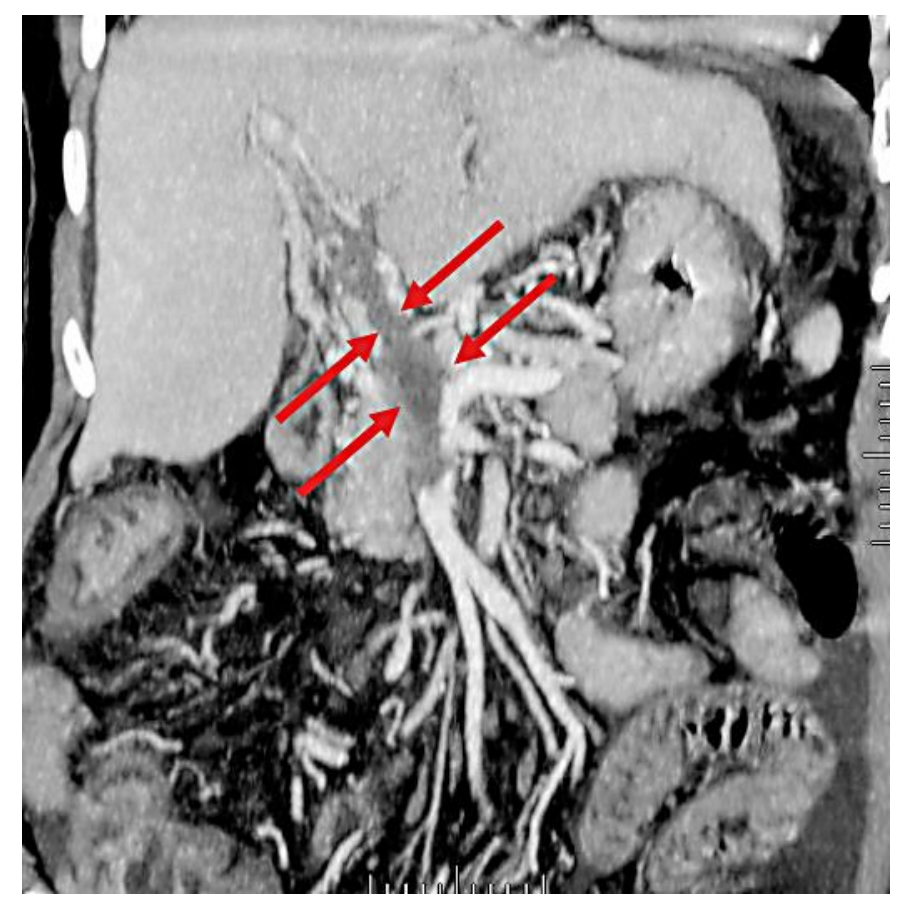

Fig. 2. A CTA showed a portal vein thrombus (black area) and a normal superior mesenteric vein in 2008.

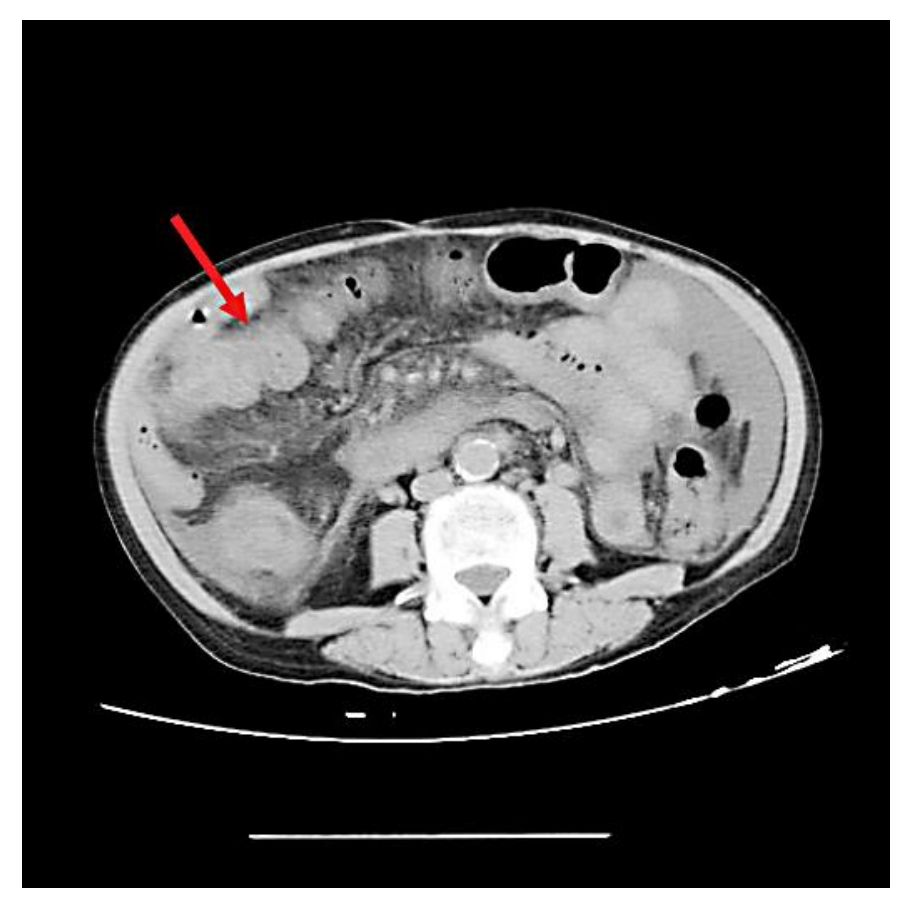

Fig. 3. An enhancement CT showed mesenteric ischemia (intestinal dilatation) in 2008. 


\begin{tabular}{|c|c|c|}
\hline \multirow{2}{*}{$\begin{array}{l}\text { Case Reports in } \\
\text { Nephrology } \\
\text { and Dialysis }\end{array}$} & \multicolumn{2}{|c|}{ Case Rep Nephrol Dial 2015;5:145-151 } \\
\hline & DOI: $10.1159 / 000431084$ & $\begin{array}{l}\text { ( ) } 2015 \text { S. Karger AG, Basel } \\
\text { www.karger.com/cnd }\end{array}$ \\
\hline
\end{tabular}

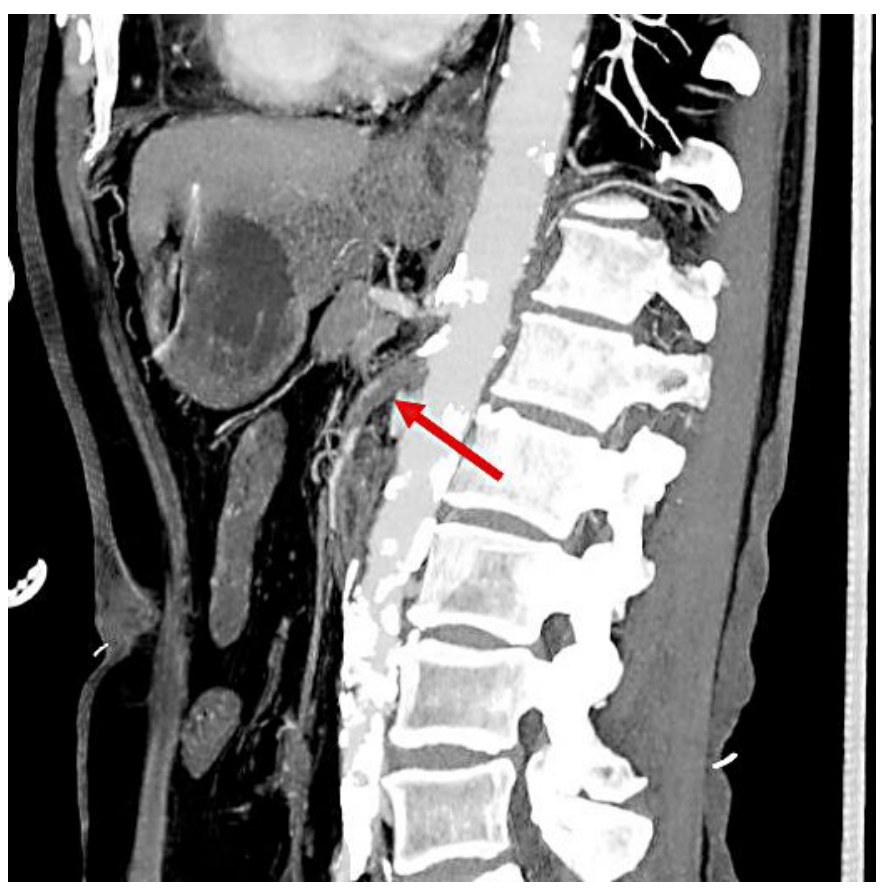

Fig. 4. A CTA showed mesenteric artery thrombosis and abdominal aorta calcification in 2008. 